This is an electronic reprint of the original article. This reprint may differ from the original in pagination and typographic detail.

\author{
Author(s): Volchenko, V.; Volchenko, G.; Akhrameev, E.; Bezrukov, L.; Dzaparova, I.; Enqvist, T.; \\ Inzhechik, L.; Izmaylov, A.; Joutsenvaara, J.; Khabibullin, M.; Khotjantsev, A.; Kudenko, \\ Yu.; Kuusiniemi, P.; Lubsandorzhiev, B.; Mineev, O.; Petkov, V.; Poleshuk, R.; \\ Shaibonov, B.; Sarkamo, J.; Shaykhiev, A.; Trzaska, Wladyslaw; Yanin, A.; Yershov, and \\ N.
}

Title: The features of electronics structure of the multichannel scintillation module for the EMMA experiment

Year: $\quad 2011$

Version:

Please cite the original version:

Volchenko, V., Volchenko, G., Akhrameev, E., Bezrukov, L., Dzaparova, I., Enqvist, T., Inzhechik, L., Izmaylov, A., Joutsenvaara, J., Khabibullin, M., Khotjantsev, A., Kudenko, Y., Kuusiniemi, P., Lubsandorzhiev, B., Mineev, O., Petkov, V., Poleshuk, R., Shaibonov, B., Sarkamo, J., . . Yershov, A. N. (2011). The features of electronics structure of the multichannel scintillation module for the EMMA experiment. Astrophysics and Space Sciences Transactions, 7(2), 171-174. https://doi.org/10.5194/astra-7-171-2011

All material supplied via JYX is protected by copyright and other intellectual property rights, and duplication or sale of all or part of any of the repository collections is not permitted, except that material may be duplicated by you for your research use or educational purposes in electronic or print form. You must obtain permission for any other use. Electronic or print copies may not be offered, whether for sale or otherwise to anyone who is not an authorised user. 


\title{
The features of electronics structure of the multichannel scintillation module for the EMMA experiment
}

\author{
V. Volchenko ${ }^{1}$, G. Volchenko ${ }^{1}$, E. Akhrameev ${ }^{1}$, L. Bezrukov ${ }^{1}$, I. Dzaparova ${ }^{1}$, T. Enqvist ${ }^{2}$, L. Inzhechik $^{1}$, A. Izmaylov $^{1}$, \\ J. Joutsenvaara ${ }^{2}$, M. Khabibullin ${ }^{1}$, A. Khotjantsev ${ }^{1}$, Yu. Kudenko ${ }^{1}$, P. Kuusiniemi ${ }^{2}$, B. Lubsandorzhiev ${ }^{1}$, O. Mineev ${ }^{1}$, \\ V. Petkov ${ }^{1}$, R. Poleshuk ${ }^{1}$, B. Shaibonov ${ }^{1}$, J. Sarkamo $^{2}$, A. Shaykhiev ${ }^{1}$, W. Trzaska ${ }^{3}$, A. Yanin ${ }^{1}$, and N. Yershov ${ }^{1}$ \\ ${ }^{1}$ Institute for Nuclear Research of RAS, Moscow, Russia \\ ${ }^{2}$ CUPP/Pyhasalmi, University of Oulu, Oulu, Finland \\ ${ }^{3}$ Department of Physics, University of Jyvaskyla, Jyvaskyla, Finland
}

Received: 20 October 2010 - Revised: 17 January 2011 - Accepted: 19 January 2011 - Published: 9 May 2011

\begin{abstract}
A brief description of the developed structural electric diagrams of 16-channel scintillation module for the underground EMMA experiment, the basic characteristics and parameters of the electrical diagrams of this module are presented. Multi-pixel photodiodes operating in a limited Geiger mode are used for photoreadout of the scintillator detectors in 16-channel scintillation module. The method of the automatic tuning of the photosensors gain based on the stabilization of an average counting rate of the scintillation detectors from gamma rays of a natural radioactive background is described.
\end{abstract}

\section{Introduction}

The EMMA experiment is under construction in the Pyhasalmi mine in central Finland. The aim of the experiment is to study the primary cosmic rays chemical composition at and above the "knee" region of $\sim 3 \times 10^{15} \mathrm{eV}$ by measuring underground cosmic ray muons multiplicity and their lateral distribution and arrival direction (Enqvist et al., 2008, 2007). The EMMA detector consists of drift chambers and plastic scintillator detectors and has the total area of $\sim 135 \mathrm{~m}^{2}$ at the depth of $85 \mathrm{~m}$ (about $240 \mathrm{~m}$ of water equivalent). In total, 1536 scintillation detectors, $122 \times 122 \mathrm{~mm}^{2}$ and $3 \mathrm{~cm}$ thick, are arranged in $4 \times 4$ arrays, each of 16 counters, which form individual modules of $50 \mathrm{~cm} \times 50 \mathrm{~cm}$ in cross section (scintillation module SC-16 or module SC-16).

The scintillator detector (it has been in detail described in works (Akhrameev et al., 2009; Bezrukov et al., 2010) has

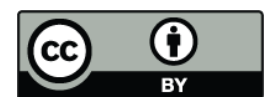

Correspondence to: V. Volchenko (vvolchenko@yandex.ru) wavelength shifting (WLS) fiber readout. One of the fiber ends is connected with a multi-pixel avalanche photodiode with a metal-resistor-semiconductor layer structure operating in a limited Geiger mode (hereafter referred to as MRS APD or APD) which is used for photoreadout of the scintillator detector. The first measurements carried out with the scintillator detector in the surface and underground laboratories demonstrate its high cosmic ray muon detection and background rejection efficiencies (Akhrameev et al., 2009; Volchenko et al., 2010).

The module SC-16 consists of 16 optically isolated scintillation detectors with WLS fiber and photosensitive MRS APD and module electronics. The electronics structure of the module SC-16 is described in this paper.

\section{The functional diagram of electrical units of the scintillation module SC-16}

The circuit (Fig. 1) works in the following way: the pulse signals generated by photosensors from each of 16 scintillation detectors come to symmetric differential inputs of broadband amplifiers (A1 - A16) that allow to provide a high noise immunity of the each channel. The amplified signals by broadband amplifiers come to high-speed discriminators (D1 - D16) and the analog adder $\left(\sum A\right)$.The discriminators are used for the formation of the signals fired detectors located in the module and the timing to the leading edge of the APD pulse.

The generated by discriminators pulses are branched and entered to the digitizer and the logic adder $\left(\sum L\right)$ where the function of a disjunction is carried out. The first fired photosensor provides the formation of the leading edge of the pulse

Published by Copernicus Publications on behalf of the Arbeitsgemeinschaft Extraterrestrische Forschung e.V. 


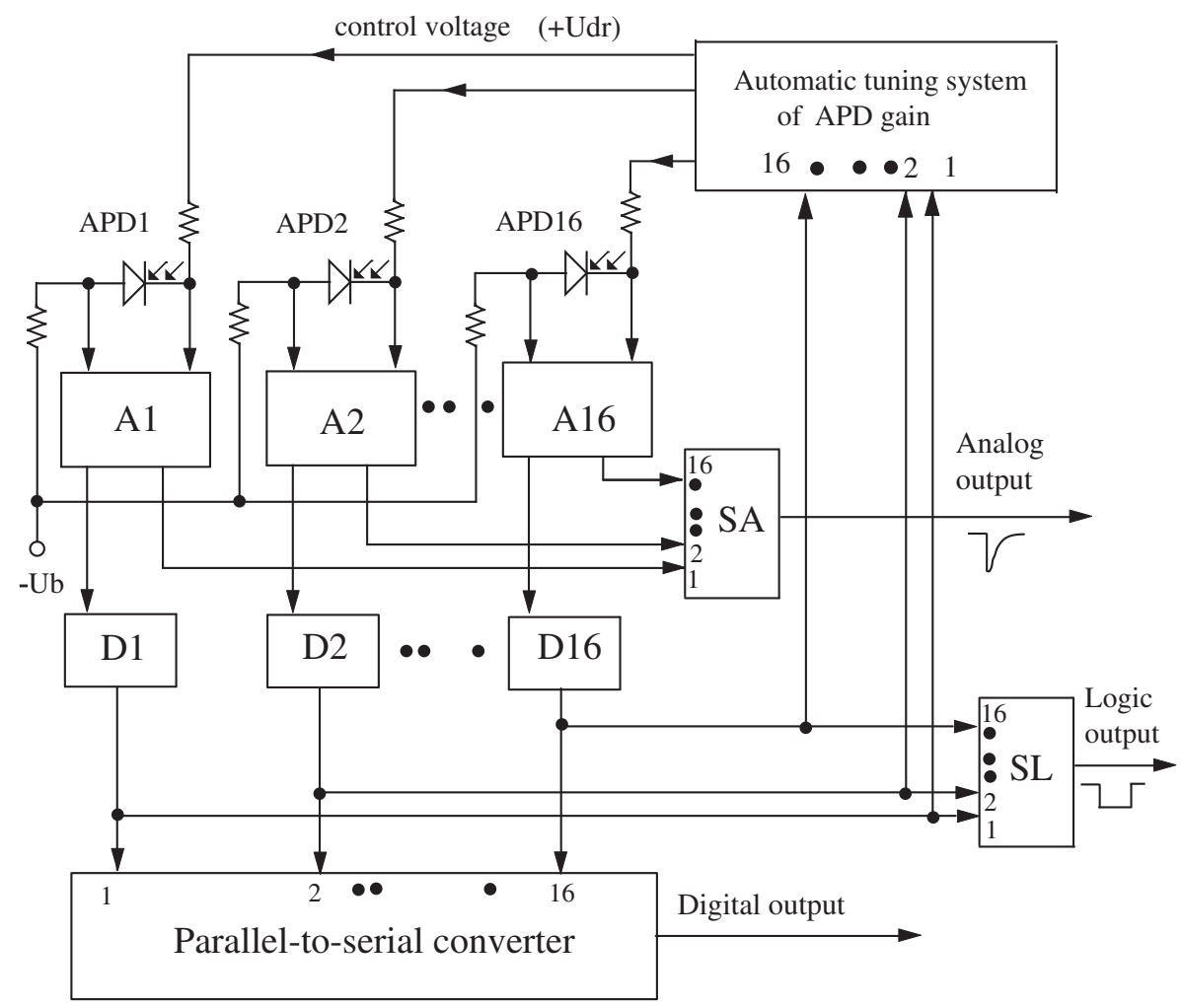

Fig. 1. The functional diagram of the electrical units of 16-channel scintillation module.

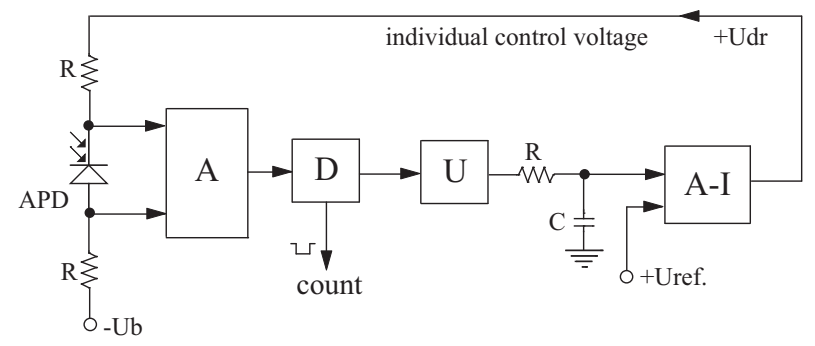

Fig. 2. The simplified functional diagram of the automatic tuning of the photosensor gain.

at the logic adder output which is taken as the time mark of the module operation.

The logic pulse having the duration of $100 \mathrm{~ns}$ comes from the module to the forming unit of the facility trigger. Simultaneously the pulses from discriminators come to the digitizer which converts the parallel position code containing the numbers of the fired detectors to the serial code. The code signals of the module's digitizer come to the hodoscope.

The analog adder signals are used for the control and predetermined calibration of parameters of the module.

The power supply of photosensors is a common stable base voltage $(-U b)$ applied to the anode of APD and individual control voltage $(+U d r)$ applied to the cathode of APD with the purpose of automatic maintenance of the detectors sen- sitivity at the given level. The individual control voltage is produced by the automatic tuning system of the APD gain which is used in this type of detector for the first time.

\section{The simplified function diagram of the automatic tun- ing of the photosensor gain}

The method of the automatic tuning of APD gain is based on a fixing of an energy threshold of the detector to an average counting rate of gamma rays of a natural radioactive background through the effective area of the scintillation detector under the condition that given flux is sufficiently stable in the limited volume of the underground facility.

The average count rate of the detector (threshold equals to $2 \mathrm{MeV}$ ) from the gamma rays of a natural radioactive background in the Pihasalmi copper mine at the depth of $240 \mathrm{~m}$ w.e. is $\sim 5 \mathrm{~Hz}$ (the measuring result, Akhrameev et al., 2009). The registration efficiency of cosmic muons by this detector with the threshold of $2.6 \mathrm{MeV}$ exceeds $95 \%$ (Bezrukov et al., 2010). Taking into consideration the method error of measurements and process-dependent parameters spread of the electronic circuits the energy threshold of the detector has been chosen $2 \mathrm{MeV}$ for reliability control of the given efficiency.

The functional diagram of the automatic tuning of the detector to the given threshold is shown on Fig. 2. 


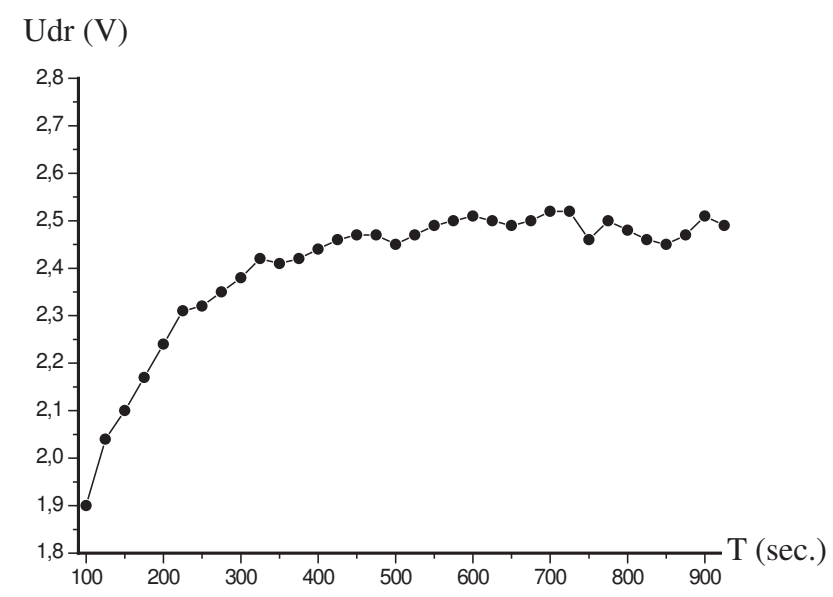

Fig. 3. Time analysis of the control voltage change in $100 \mathrm{~s}$ after the switch on of the automatic tuning.

APD pulses after preliminary amplification by the amplifier (A) come to the discriminator (D) with the fixed threshold. The discriminator forms the counting pulses and their intensity is the sensitivity index of the detector of a natural radioactive background gamma ray and cosmic muons. The highly stable univibrator (U) standardizes the duration and amplitude of these pulses and then they are integrated by the $\mathrm{RC}$ circuit for the generation of the voltage which is proportional to the average operation frequency of the discriminator. The timing elements of the univibrator are chosen in such a manner that only at the given average counting rate of pulses $(\sim 5 \mathrm{~Hz})$ the equality of the integrated voltage and the given reference voltage (Uref) is achieved and the output voltage $(+U d r)$ of the amplifier-integrator (A-I) remains without change (it equals to $2.5 \mathrm{~V}$ ). Sum of the positive output voltage of the amplifier-integrator $(+U d r)$ applied to the APD cathode and the base negative voltage $(-U b)$ applied to the anode of APD determines the APD gain. When the count rate of the detector has deviation from set value the output voltage of the amplifier-integrator changes and the APD gain changes too and its sign is always the opposite to the change of the count rate of the detector. Thus, the circuit of the negative feedback is closed and the whole scheme converts into the compensating stabilizer for which the stabilized parameter is an average counting rate of the detector and the APD gain bound to it.

As can be seen from Fig. 3, the setup time of the control voltage $(+U d r)$ is $600-700 \mathrm{~s}$ after the switch on of the automatic tuning scheme.

Figure 4 shows energy-release spectra for the same scintillation detector: with and without automatic tuning of the APD gain. Spectra are identical, that allows using the mode of binding of the energy-release in the scintillator from the cosmic muons for the continuous work and also for the control and calibration works of the detector.

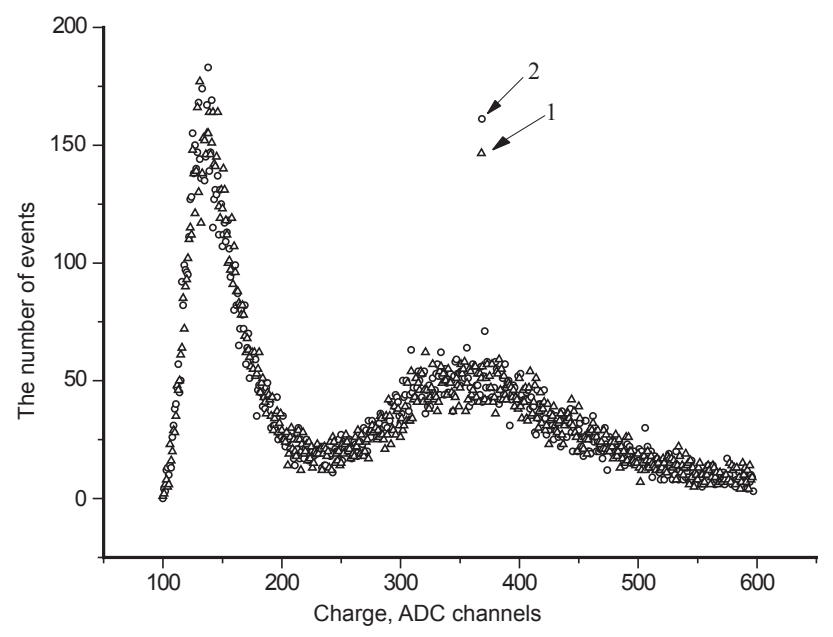

Fig. 4. Energy-release spectra of the scintillation detector: 1 - with and 2 - without automatic tuning of APD gain. Time of measurement equals to $4000 \mathrm{~s}$. Threshold of the detector equals to $2 \mathrm{MeV}$. Average count rate of events equals to $4.95 \mathrm{~Hz}$.

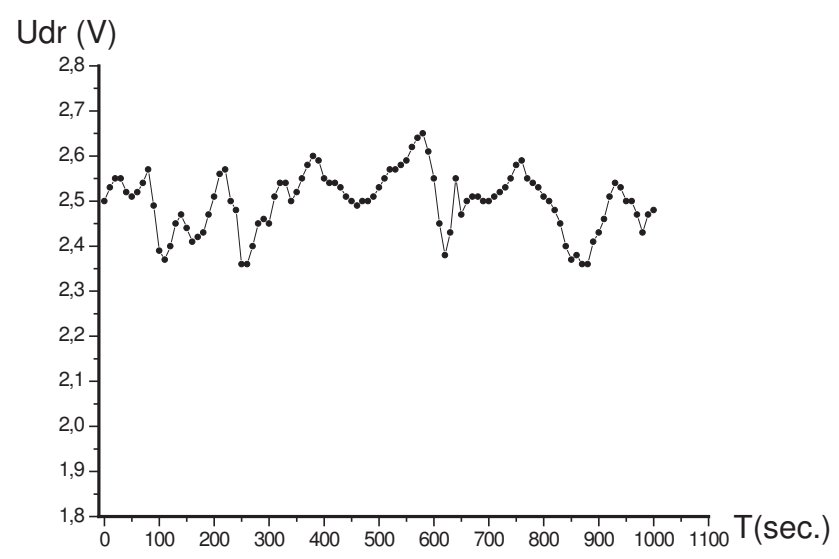

Fig. 5. Control voltage fluctuations in the stabilization mode of the APD sensitivity.

The characteristic fragment of time fluctuations of the control voltage from the diagram of observation during one hour is obtained (see Fig. 5). Such fluctuations of the control voltage are characteristic for any photosensor. The fluctuations connected with the response of the inertial system of the stabilization to the irregular character of signals are essentially unavoidable. The measurements show that the standard deviation of the control voltage equals to $0.067 \mathrm{~V}$ at the average value of the control voltage $(2.5 \mathrm{~V})$ and it equals to $0.22 \%$ with respect to the APD total supply voltage $(\sim 30 \mathrm{~V})$.

If the variation of APD gain from the supply voltage is $\sim 3.6 \%$ on every $0.1 \mathrm{~V}$ the standard deviation of APD gain equals to $2.4 \%$.

The temperature instability of average frequency $(\mathrm{f} \sim 5 \mathrm{~Hz})$ of the electronic scheme no more than $0.12 \% /{ }^{\circ} \mathrm{C}$.

The frequency deviations don't exceed $\pm 0.05 \%$ at the variations of the supply voltage of the electronic circuit on $\pm 10 \%$. 


\section{Conclusions}

The principal advantage of the electronic scheme applied in the module is its ability to automatically adjust of everyone scintillation detector to the given energy threshold by intensity of a natural radioactive background gamma ray in the mine and to keep the threshold at the set level (under the influence of destabilizing factors). 1536 scintillation detectors are placed in 96 modules of the EMMA facility therefore the individual adjustment of the sensitivity of each detector under condition of variations of APD gain and scintillator specific light yield is impossible. The original technique of automatic tuning of an energy threshold is suitable for any type of nuclear radiation detectors. This technique is used for the first time.

Edited by: T. Suomijarvi

Reviewed by: two anonymous referees

\section{References}

Akhrameev, E. V., Bezrukov, L. B., Dzaparova, I. M., et al. Multi-pixel Geiger-mode avalanche photodiode and wavelengthshifting fibre-optics readout of plastic scintillator counters for the EMMA underground experiment, Nucl. Instr. Meth. Physics Res. A, 610, 419-422, 2009.

Bezrukov, L., Butin, K., Davitashvili, I., et al.: Geiger mode APD's for the underground cosmic ray experiment EMMA, Proc. Science, PD09:026, 2010.

Enqvist, T., Joutsenvaara, J., Jamsen, T., et al.: Underground cosmic-ray experiment EMMA, Nucl. Physics B, 165, 349 - 354 2007.

Enqvist, T., Joutsenvaara, J., Jamsen, T., et al.: EMMA - a new underground cosmic-ray experiment, Nucl. Physics B, 175 - 176, 307-310, 2008.

Volchenko, V. I., Akhramev, E. V., Bezrukov, L. B., et al.: Background and muon counting rates in underground muon measurements with a plastic scintillator counter based on a wavelength shifting fibre and a multi-pixel avalanche photodiode readout, Central Europ. J. Physics, 8, 445 - 447, 2010. 